\title{
Successful Treatment of Crizotinib-Associated Severe Hepatotoxicity in Anaplastic Lymphoma Kinase-Positive Non-Small Cell Lung Cancer
}

\author{
Joon Jin', Seo-Yeon Ahn', Sunmin Kim ', Ho-Sung Lee', In-Jae Oh ${ }^{1,2}$, Kyu-Sik Kim ${ }^{1,2}$, Young-Chul Kim ${ }^{1,2}$, Yoo-Duk Choi ${ }^{2,3}$ \\ 'Department of Internal Medicine, Chonnam National University Medical School, Gwangju; ${ }^{2}$ Lung and Esophageal Cancer Clinic, Chonnam National University \\ Hwasun Hospital, Hwasun; ${ }^{3}$ Department of Pathology, Chonnam National University Medical School, Gwangju, Korea
}

\begin{abstract}
Crizotinib-associated severe hepatotoxicity has been rarely reported and experts recommended stopping crizotinib treatment in patients with grade $3 / 4$ transaminase elevation. We experienced a case of anaplastic lymphoma kinase-positive non-small cell lung cancer occurring as a result of severe hepatotoxicity due to crizotinib-associated hepatitis, accompanied by the reactivation of chronic hepatitis B, which was reversed with dose reduction and anti-viral therapy. Our case highlights the possibility that crizotinib might induce hepatitis and this might be associated with the underlying presence of chronic hepatitis B. In addition, crizotinib could be continued with reduced unless there are any other therapeutic options.
\end{abstract}

Keywords: Crizotinib; EML4-ALK fusion protein, human; Hepatitis

\section{INTRODUCTION}

Crizotinib is an orally active, small-molecule tyrosine kinase inhibitor (TKI) of anaplastic lymphoma kinase (ALK) that is efficacious against non-small cell lung cancer (NSCLC) with echinoderm microtubule-associated protein-like 4 (EML4)-ALK rearrangement [1]. Common adverse events include visual disturbance, nausea a, vomiting, diarrhea, peripheral edema, constipation, and fatigue [2]. Crizotinib-associated severe grade hepatotoxicity has been rarely reported [3] and the drug should be discontinued if grade 3/4 elevations of transaminases were shown. Herein, we report one case with ALK-positive NSCLC who developed severe hepatotoxicity due to crizotinib-associated hepatitis combined with reactivation of chronic hepatitis $B$ after crizotinib medication and was reversible with dose reduction and anti-viral therapy.

\section{CASE REPORT}

A 51-year-old woman with no past smoking history was admitted to us with chief complaints of dyspnea and hoarseness. The patient had a past medical history of hypertension and chronic hepatitis B carrier. Because of normal hepatic functions, the patient was under regular follow-up without anti-viral therapy at an outside hospital. In addition, the patient had a 2-month-history diagnosis of stage IV (T2a N3 M1b) adenocarcinoma without epidermal growth factor receptor mutation, for which the patient received two cycles of first-line chemotherapy with pemetrexed 500 $\mathrm{mg} / \mathrm{m}^{2}$ and cisplatin $60 \mathrm{mg} / \mathrm{m}^{2}$ at a 3-week interval.

On physical examination, the patient presented with swollen face, neck vein distension and inspiratory stridor. But this was not accompanied by jaundice or hepatomegaly. Liver function tests showed aspartate aminotransferase (AST) $335 \mathrm{U} / \mathrm{L}$ and alanine aminotransferase (ALT) 599 U/L. Hepatitis Bs antigen and hepatitis Be antibody were positive. Hepatitis Be antigen, hepatitis $\mathrm{C}$ antibody and Hepatitis A immunoglobulin $\mathrm{M}$ antibody were negative. Hepatitis B virus (HBV) DNA concentration was 1,690,000 IU/mL. The patient had no history of taking other hepatotoxic drugs or herbal medications. We assumed that chronic hepatitis $\mathrm{B}$ was reactivated in our case. We therefore started to administer lamivudine $100 \mathrm{mg}$ once daily to the patient.
Correspondence to: In-Jae Oh

Department of Internal Medicine, Chonnam National University Hwasun Hospital, 322 Seoyang-ro, Hwasun 519-763, Korea

Tel: +82-61-379-7617, Fax: +82-61-379-7619, E-mail: droij@chonnam.ac.kr

Received: Mar 19, 2014 / Accepted after revision: Apr. 30, 2014
(C) 2014 Soonchunhyang Medical Research Institute This is an Open Access article distributed under the terms of the Creative Commons Attribution Non-Commercial License (http://creativecommons.org/licenses/by-nc/3.0/) 

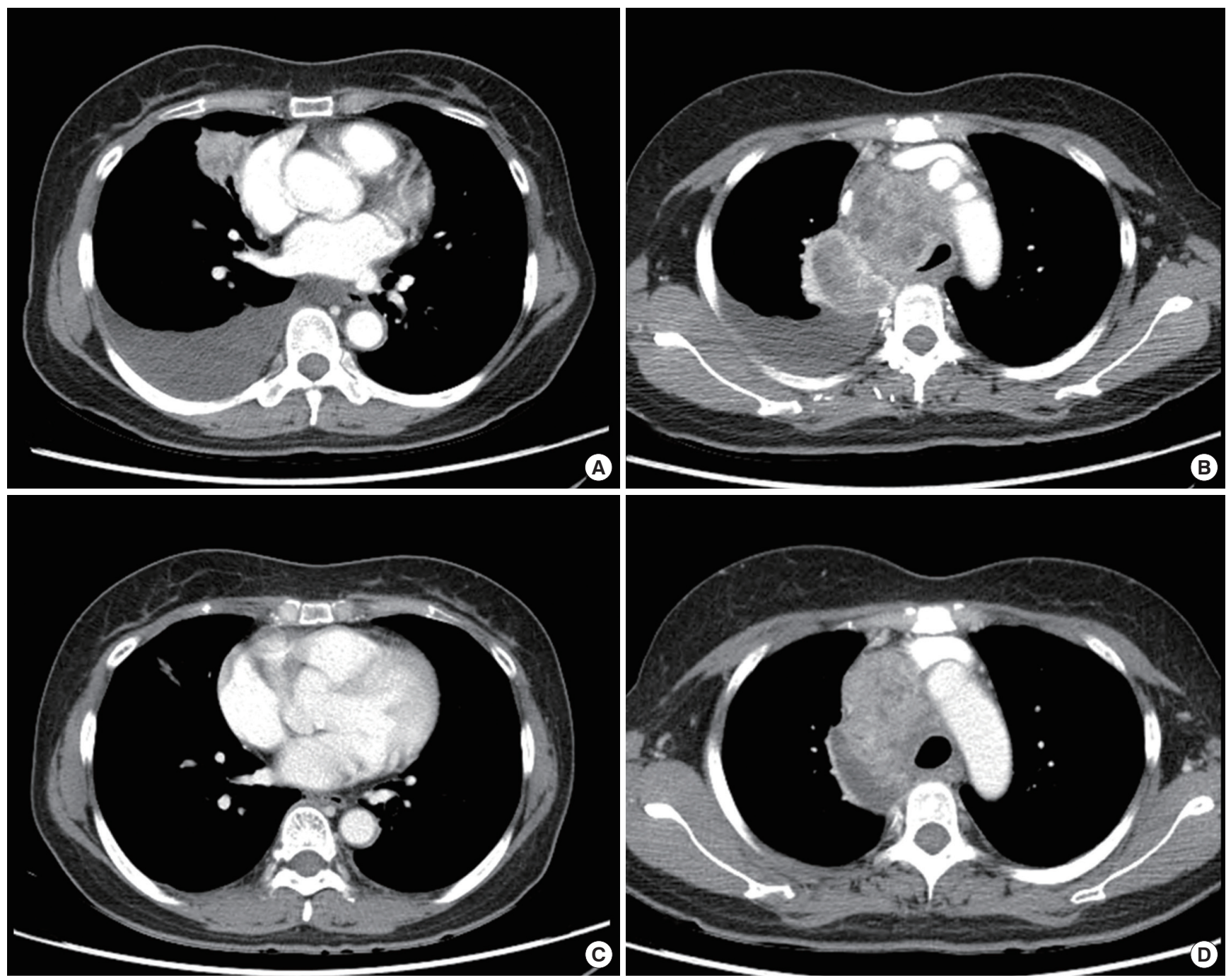

Fig. 1. Chest computed tomography (CT) on admission. (A) Chest CT scans on admission show a primary tumor in right middle lobe and moderate amount of pleural effusion. (B) Huge mediastinal lymphadenopathies compress trachea and superior vena cava. (C) Eleven weeks after crizotinib treatment, chest CT scans show marked resolution of tumor and (D) partial regression of mediastinal lymphadenopathies.

Chest computed tomography (CT) scans showed a stabilization of primary lung malignancy in the right middle lobe, accompanied by a moderate amount of right pleural effusion and more aggravated metastatic lymphadenopathies. This caused the tracheal shift to the left and the compression of superior vena cava (Fig. 1A, B). Five days after lamivudine treatment, the patient had a decrease in serum ALT level to $269 \mathrm{U} / \mathrm{L}$. However, the respiratory symptoms were aggravated. The patient had a positive immunohistochemistry for ALK (2+) and positive fluorescent in situ hybridization for EML4-ALK rearrangement (Fig. 2). We therefore administered crizotinib to the patient at a decreased dose $(250 \mathrm{mg}$ once daily) as a second-line treatment.

Eight days after crizotinib treatment, the patient achieved a gradual improvement of respiratory symptoms but had an increase in serum ALT level to $805 \mathrm{U} / \mathrm{L}$ (Fig. 3). We therefore determined to lower the dose of crizotinib to $250 \mathrm{mg} /$ day at a 3-day interval considering that our patient exhibited crizotinib-associated hepatotoxicity. We also continued lamivudine treatment for the management of reactivation of chronic hepatitis B. Eight days after the reduction of dosage, the patient had a decrease in serum ALT level to $200 \mathrm{U} / \mathrm{L}$. Then, we elevated the dose of crizotinib to the standard one of $250 \mathrm{mg}$ twice a day. We also recommended switching from lamivudine to tenofovir for the patient. Eleven weeks after crizotinib treatment, the patient achieved a marked improvement of respiratory symptoms and that of serum ALT level to $57 \mathrm{U} / \mathrm{L}$. On follow-up chest CT scans, the patient achieved a 

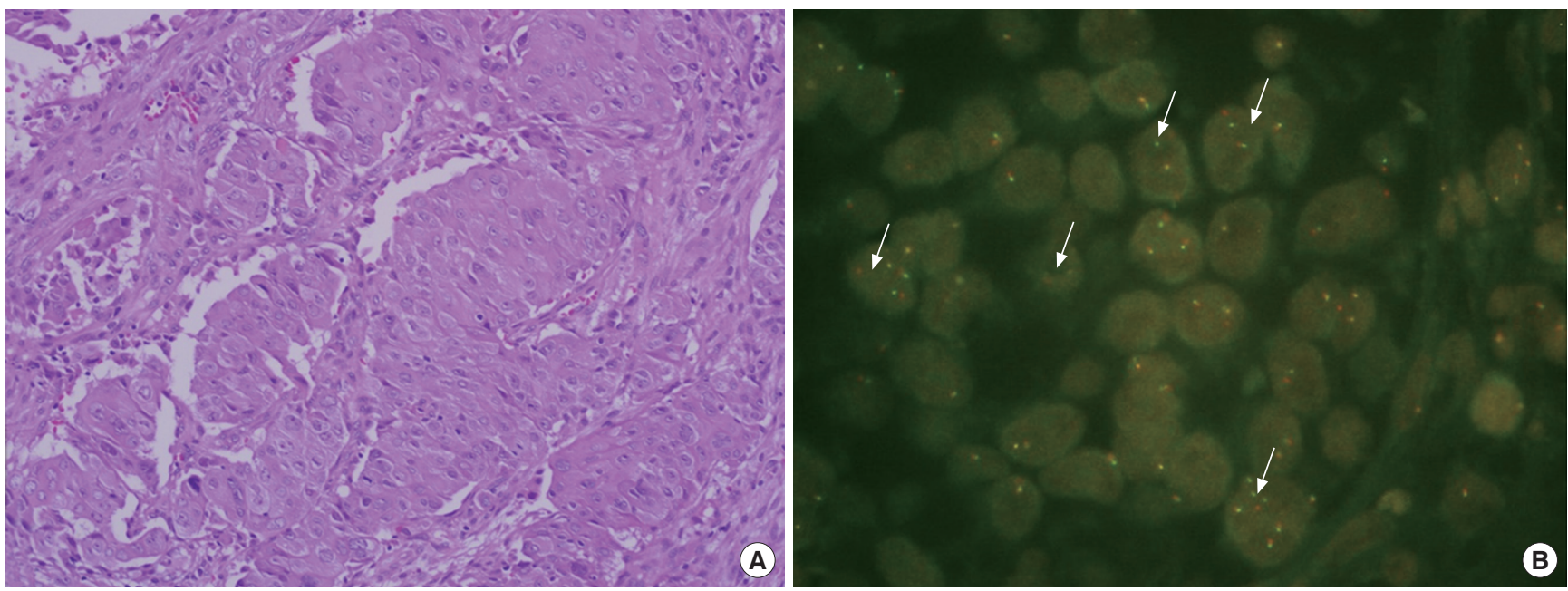

Fig. 2. Histopathology and immunohistochemistry. (A) Tumor cell shows round nuclei, prominent nucleoli and mucin in the abundant cytoplasm; these findings are suggestive of poorly differentiated adenocarcinoma (H\&E, $\times 100$ ). (B) Fluorescent in situ hybridization shows distinct and separated red and green signal (arrow); these findings are suggestive of positive immunohistochemistry for anaplastic lymphoma kinase (ALK) rearrangement (split 3' ALK-5' ALK).

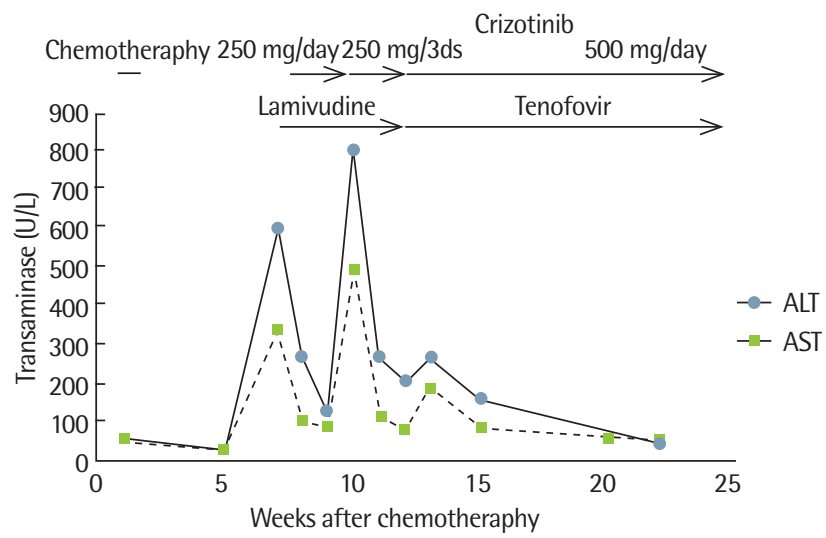

Fig. 3. Clinical course of hepatic function. Markedly elevated transaminase levels were improved with anti-viral therapy and reduced dose crizotinib treatment. Chemotherapy means second cycle of pemetrexed and cisplatin. Crizotinib 250 mg/3ds means 250 mg/day at 3-day interval. ALT, alanine aminotransferase; ALT, aspartate aminotransferase.

partial remission of primary tumor accompanied by metastatic mediastinal lymphadenopathies (Fig. 1C, D).

\section{DISCUSSION}

To our knowledge, our case is the first one of crizotinib-associated hepatitis that was successfully treated in a patient with preexisting chronic hepatitis B. Although Ripault et al. [3] previously reported a patient who developed acute hepatitis after crizotinib therapy, there was a relapse after reintroduction with reduced dose. In our case, the patient underwent tumor progression very rapidly. Therefore we could not wait until the patient achieved an improvement of liver functions. Moreover, our case is also noteworthy in that we could successfully treat it by lowering the dose of crizotinib while continuing the anti-viral therapy for the management of chronic hepatitis $\mathrm{B}$.

Recent studies have shown that the response rate was $50 \%$ to $61 \%$ following crizotinib treatment in patients with NSCLC with ALK rearrangement $[2,4]$. Phase I and II clinical studies have shown that serum ALT elevation was noted in 15\% (grade 3/4, 7\%) and of AST in $11 \%$ (grade $3 / 4,3 \%$ ) of patients receiving crizotinib $[2,5]$. Most cases of serum ALT elevation are usually reversible. In addition, patients with serum ALT elevation due to crizotinib therapy may be given the treatment again at the same or decreased dose. But total bilirubin elevation after crizotinib therapy has been described less commonly [6].

Still, little is known about exact mechanisms by which TKI-associated hepatotoxicity occurs [7]. To date, two hypothetic mechanisms have been proposed to explain its cause as shown below: 1) the reactive metabolites are produced once crizotinib is metabolized. These metabolites can interfere with cellular molecules and thereby impair the cellular functions, thus causing cell death [810]. Moreover, they have also been suggested to be associated with other types of idiosyncratic hepatotoxicity [11]. 2) As the immunemediated mechanism $[8,12]$, the initial immunosuppressive stage is characterized by a marked increase in serum levels of $\mathrm{HBV}$ DNA, hepatitis Be antigen and HBV DNA polymerase. This stage is probably related to the suppression of immune mechanism that serves to control HBV replication. The immune-restoration stage 
occurs following withdrawal of cytotoxic or immunosuppressive drugs, resulting in a rapid immune-mediated destruction of infected hepatocytes. This may be followed by fatal clinical conditions such as hepatitis, hepatic failure and even death.

Our case highlights the possibility that crizotinib might induce hepatitis and this might be associated with the underlying presence of chronic hepatitis B. In our case, grade 4 hepatic dysfunction was developed after two cycles of chemotherapy and gradually improved after receiving the anti-viral agent for HBV. Although the HBV load was not examined before chemotherapy, serum levels of AST and ALT were normalized before treatment. In addition, high level of HBV load was detected at the time of hepatic flare. It would be rational to consider reactivation of $\mathrm{HBV}$ as the cause of hepatic flare. In addition, we assumed that the patient was suspected of having crizotinib-induced hepatitis based on rapid re-elevation of serum ALT level after the initiation of crizotinib at a reduced dose. It remains unclear, however, whether preexisting chronic HBV is a risk factor of developing crizotinib-associated hepatitis in patients receiving conventional chemotherapy. Although early studies showed that prophylatic use of lamivudine was effective for patient with chemotherapy-induced HBV reactivation, the effect of lamivudine, starting after clinical evidence of hepatitis, is not fully established and long-term treatment may induce the YMDD mutation, which can cause hepatitis flare [13]. Thus, agent with more favorable resistance profile, such as tenofovir or entecavir is preferred like our case [14].

In conclusion, our case indicates that the preexisting chronic hepatitis B may be a risk factor of developing crizotinib-associated hepatitis and reactivating HBV in patients with NSCLC with ALK rearrangement. Clinicians should be aware of the possibility of severe crizotinib-associated hepatotoxicity and monitor the hepatic functions. In addition, they should also consider lower the dose of crizotinib unless there are any other therapeutic options.

\section{REFERENCES}

1. Gerlinger M, Norton L, Swanton C. Acquired resistance to crizotinib from a mutation in CD74-ROS1. N Engl J Med 2013;369:1172-3.

2. Camidge DR, Bang YJ, Kwak EL, Iafrate AJ, Varella-Garcia M, Fox SB, et al. Activity and safety of crizotinib in patients with ALK-positive nonsmall-cell lung cancer: updated results from a phase 1 study. Lancet Oncol 2012;13:1011-9.

3. Ripault MP, Pinzani V, Fayolle V, Pageaux GP, Larrey D. Crizotinib-induced acute hepatitis: first case with relapse after reintroduction with reduced dose. Clin Res Hepatol Gastroenterol 2013;37:e21-3.

4. Ou SH. Crizotinib: a novel and first-in-class multitargeted tyrosine kinase inhibitor for the treatment of anaplastic lymphoma kinase rearranged non-small cell lung cancer and beyond. Drug Des Devel Ther 2011;5:471-85.

5. Rothschild SI, Gautschi O. Crizotinib in the treatment of non-small-cell lung cancer. Clin Lung Cancer 2013;14:473-80.

6. Teo YL, Ho HK, Chan A. Risk of tyrosine kinase inhibitors-induced hepatotoxicity in cancer patients: a meta-analysis. Cancer Treat Rev 2013;39: 199-206.

7. Navarro VJ, Senior JR. Drug-related hepatotoxicity. N Engl J Med 2006; 354:731-9.

8. Li X, Kamenecka TM, Cameron MD. Bioactivation of the epidermal growth factor receptor inhibitor gefitinib: implications for pulmonary and hepatic toxicities. Chem Res Toxicol 2009;22:1736-42.

9. Li X, Kamenecka TM, Cameron MD. Cytochrome P450-mediated bioactivation of the epidermal growth factor receptor inhibitor erlotinib to a reactive electrophile. Drug Metab Dispos 2010;38:1238-45.

10. Teng WC, Oh JW, New LS, Wahlin MD, Nelson SD, Ho HK, et al. Mechanism-based inactivation of cytochrome P450 3A4 by lapatinib. Mol Pharmacol 2010;78:693-703.

11. Ju C, Uetrecht JP. Mechanism of idiosyncratic drug reactions: reactive metabolite formation, protein binding and the regulation of the immune system. Curr Drug Metab 2002;3:367-77.

12. Xunrong L, Yan AW, Liang R, Lau GK. Hepatitis B virus (HBV) reactivation after cytotoxic or immunosuppressive therapy: pathogenesis and management. Rev Med Virol 2001;11:287-99.

13. Gutfreund KS, Williams M, George R, Bain VG, Ma MM, Yoshida EM, et al. Genotypic succession of mutations of the hepatitis B virus polymerase associated with lamivudine resistance. J Hepatol 2000;33:469-75.

14. Lok AS, McMahon BJ. Chronic hepatitis B: update 2009. Hepatology 2009;50:661-2. 